\title{
El cuidado real frente a la vulnerabilidad del estudiante de enfermería
}

\author{
Real health care in the face of the vulnerability of the nursing student \\ Betsy Corina Sosa-García ${ }^{1,2}$ orcid.org/0000-0002-1545-4190 \\ Cleotilde García-Reza1,3 orcid.org/0000-0002-6598-8617 \\ Mauricio Arias-Rojas4* orcid.org/0000-0003-2096-1792
}

1. Facultad de Enfermería y Obstetricia, Universidad Autónoma del Estado de México. Toluca, México.

2. Cuerpo de enfermería y cuidado a la salud. Universidad Autónoma del Estado de México. Toluca, México.

3. Equipo de enfermería y atención médica. Universidad Autónoma del Estado de México. Toluca, México.

4. Facultad de Enfermería, Universidad de Antioquia. Medellín, Colombia.

\section{Resumen}

Introducción: El intercambio de experiencias con pares en la práctica diaria permite al estudiante el aprendizaje del cuidado de enfermería. Objetivo: Comprender las representaciones sociales del cuidado, construidas desde el cotidiano del estudiante de enfermería mexicano. Materiales y métodos: Estudio cualitativo, basado en la teoría de la representación social en 46 estudiantes que realizaban prácticas clínicas, a quienes se les aplicó entrevista semiestructurada, que se analizó a través de la agrupación de categorías empíricas que sustentan la composición de los resultados subsecuentes, comparando los datos con relatos de los informantes. Resultados: La concepción del proceso de cuidado se estructura con rangos que representan lo social de los entrevistados durante su formación, la cual los torna vulnerables en dicho proceso. Las vivencias se construyen y reconstruyen en su afán por descubrir sus experiencias en el cuidar para dar respuesta a los desafíos del ser. Sin embargo, en estas experien cias se altera su mundo, irrumpiendo la paz mental. Conclusiones: Los procesos de interacción posiciona al estudiante frente al mundo social del cuidado a partir de bases filosóficas, a fin de reflexionar con su ser y hacer, acción que orienta sus conductas en el cotidiano del cuidado del otro.

Palabras clave: Cuidado preventivo; estudiantes de enfermería; investigación cualitativa; investigación en enfermería. (Fuente: DeCS, Bireme).

\begin{abstract}
Introduction: The exchange of daily practicing experiences with peers is useful for students to learn about nursing care. Objective: To understand the social representations of health care that are constructed from the daily life experiences of the Mexican nursing student. Materials and methods: A qualitative study based on the social representation theory with 46 students who carried out clinical practices. A semi-structured interview was applied, which was analyzed through grouping of empirical categories that support the composition of the subsequent results, comparing the data with reports from the participants. Results: The conception of the health care process is structured with ranges that represent the social aspects of the students during their training, which in turn makes them vulnerable. The experiences are constructed and re-constructed as a consequence of the students' eagerness to discover their experiences in health care in order to respond to their challenges. However, these experiences affect the students' world, disrupting their peace of mind. Conclusions: Interaction processes position students in the face of the social world of health care based on philosophical bases in order to reflect with their being and doing, an action that guides their behavior in the daily care of the other.
\end{abstract}

Keywords: Care; nursing students; qualitative research; nursing research. (Source: DeCS, Bireme).

\footnotetext{
*Autor de correspondencia

Mauricio Arias-Rojas

e-mail: emauricio.arias@udea.edu.co
} 


\section{Introducción}

La experiencia de cuidado de los estudiantes de enfermería, durante su proceso de formación profesional; representa un reto social único que involucra el cuidado en estos contextos(1,2). Este cuidado se construye a partir de experiencias, información, conocimientos y modelos de pensamiento que reciben(2,3), en este sentido, la representación social del cuidado, según Moscovici(4), es un punto de interés por aprender los paradigmas sobre el cuidado humano, comprender y transformar al ser de cuidado; de esta forma se aproxima a la teoría de la representación social(5,6). Se optó por este referencial para facilitar el análisis de la citada representación de los estudiantes de enfermería frente al cuidado $(4,7)$.

Con este abordaje teórico-filosófico de las representaciones sociales $(4,5)$, se rescatan significados y experiencias subjetivas de la vida diaria de estos estudiantes $(6,8)$, aprendizaje que permea la estructura social, lugar donde se forma el ser y el hacer(2) en los diferentes contextos de su experiencia cotidiana ${ }^{(4,9)}$; en otras palabras pueden ser una expresión del conocimiento del sentido común. Desde esta perspectiva y de acuerdo con Jodelete(5), la aproximación de estos actores sociales a partir de sus vivencias, recuerdos y emociones, representa el cuidado ante la situación que los vulnera(5,10). En la actualidad, si bien el cuidado ha sido ampliamente estudiado, organizado y estructurado dentro de una estructura holarquica, propuesta desde el pensamiento científico; sin embargo, conocer el cuidado desde el pensamiento del sentido común de quienes están formándose en la disciplina de enfermería, aporta a la estructuración y avance del conocimiento de enfermería en un grupo específico(7).

Partiendo de esta premisa y desde el punto de vista de los estudiante de enfermería, las representaciones sociales envuelven un significado frente a la situación del estudiante en su mundo hospitalario(5); el cuidado simboliza el modo de estar con el otro ser social durante su travesía de enfermedad(11), en cuestiones especiales de la vida, para restaurar o conservar la salud, aceptar o resignarse a la muerte, situación que los posiciona en un rol social del ser y del hacer(12), al construirse durante su formación profesional(2,12).

En este rol, las vivencias, significan descubrir las relacionadas con el cuidar del otro(3,13), para responder a los desafíos de intercambio de experiencias, con sus pares y con su mundo social cotidiano(14,15). Bajo esta postura, las representaciones sociales se aproximan al conocimiento lego y al cotidiano en el contexto colectivo, para apropiarse de una postura filosófica sobre el cuidado y lograr con ello comprender el conocimiento científico desde las vivencias cotidianas del estudiante, hasta transformar los escenarios de cuidado durante el proceso de formación, irrumpe la paz mental(16), manifiesta por un alto sentido de estrés(6,16).

Un estudio señala que los espacios clínicos representan escenarios de aprendizaje para una praxis repetitiva y cíclica; en este sentido, el apoyo de tutores evita cometer errores(17), por cuanto al interactuar sustentan el aprendizaje desde la percepción dual: las aulas universitarias y el hospital(18). Pero, la falta de experiencia clínica y la escasa supervisión en la enseñanza, representa un reto para adquirir destrezas y habilidades en el manejo del paciente(16,19). Mendieta et al.(6), indica que la experiencia y competencias de los estudiantes giran en torno al cuidado terapéutico del paciente, al requerir un vínculo entre los docentes y estudiantes; aquí se reconoce la tensión emocional(1) siempre presente, por los nuevos saberes, para alcanzar un cuidado exitoso(6,15).

Por otro lado, un estudio de revisión enfoca las prácticas clínicas de enfermería y los aspectos subjetivos para el logro de las competencias de cuidado. Algunos aspectos señalados por los estudiantes como contradicciones entre la teoría explicada en el aula y la práctica clínica hospitalaria(17), en este punto, dicha desconexión incide de forma negativa en el modo como los estudiantes piensan sobre el cuidado; esto ocurre en las etapas iniciales de la carrera a la hora de enfrentar las tareas $y$ el trabajar para su formación profesional( $(8,16)$.

La complejidad del aprender y apropiarse de lo que es el cuidado de enfermería para los estudiantes, representa en punto de partida de la forma como conciben, imaginan, dominan y viven su propio mundo de cuidado y el del otro(5,18), así pues, bajo este referente se da sustento y se construye en el estudiante su representación social del cuidado(19). En consecuencia, este estudio se planteó como objetivo comprender las representaciones sociales del cuidado, construidas desde el cotidiano de estudiantes de enfermería mexicanos. 


\section{Materiales y métodos}

Estudio cualitativo delineado por el referencial conceptual de las representaciones sociales Moscovici(4), como apoyo en la discusión de resultados. El estudio se realizó en una facultad de enfermería de una universidad mexicana. Se seleccionó de manera intencional $(20,21)$ a 46 estudiantes de 150 que cursaban el octavo semestre y realizaban prácticas clínicas.

Se seleccionó a los participantes con los siguientes criterios: 1) inscritos en el octavo periodo lectivo 2017-2018 de la licenciatura; 2) Tener al menos un mes de experiencia en la práctica clínica de enfermería para cumplir con las actividades relacionadas; 3) Participar en el estudio de manera voluntaria. Se excluyeron los alumnos con problemas de salud, y aquellos con limitaciones de tiempo durante la recolección de datos.

Los participantes fueron de pregrado en enfermería. El plan de estudios se desarrolla en ocho periodos lectivos, en horario integral, durante el primer periodo de 8 a 15 horas; del segundo periodo en adelante, de 8 a 18 horas.

La técnica de recolección de datos utilizada fue a través de una entrevista semiestructurada(20,21), con una guía a base de preguntas a fin de obtener descripciones referentes al cuidado. Para esta actividad, los participantes fueron entrevistados, para conocer datos sociodemográficos característicos de ellos, como edad, sexo, profesión, estado civil; además, las preguntas que orientaron la investigación estuvieron relacionadas con el proceso de cuidado, en la vida cotidiana desde la representación social del estudiante.

Al presentar el proyecto, se solicitó el campo de investigación a los directivos del organismo académico; una vez aprobada la petición, se pidió a los profesores del programa curricular, su asentimiento para presentar el objetivo del proyecto e invitar a los estudiantes a participar. posteriormente, se programó las fechas para recolectar los datos. Las entrevistas se realizaron de acuerdo con la disponibilidad del estudiante, en una oficina destinada dentro de la institución educativa.

Los participantes fueron identificados de manera numérica, conforme a la realización de las entrevistas, basadas en el orden en que se llevó a cabo la recolección de datos, durante el periodo comprendido entre noviembre de 2017 a mayo de 2018. Los datos fueron grabados con la aprobación de los participantes y transcritos de manera textual, para someterlos a análisis temático, a fin de acercarse a las categorías.

\section{Análisis de los datos}

El análisis temático y la codificación se alcanzaron por consenso, la discusión de los datos, fue por medio de un guión inicial de categorías temáticas ${ }^{(20,21)}$ diseñado a partir del objetivo. Al mismo tiempo, se fragmentaron los relatos en unidades básicas de registro, además se depuró la información hasta establecer un sistema organizativo, para el discurso de forma ordenada.

Dada la reiteración de información obtenida frente a las categorías temáticas, se consideró que esta se había saturado con información suficiente(20); de este modo se detuvo la realización de entrevistas adicionales. Finalmente, en la etapa de lectura y relectura fueron agrupados los datos con algunos verbatims, para establecer las categorías temáticas(21) resultantes de la estructuración y análisis realizado sobre el cuidado de estos alumnos, desde su cotidiano en el contexto de su formación académica y práctica, a la luz de la teoría de la representación social de Moscovici(4).

Para garantizar los criterios de rigor de esta investigación, los autores hicieron reuniones grupales para socializar los avances de los resultados. Además, dos de los investigadores hicieron el análisis cualitativo de la información, en los momentos en que hubo desacuerdo, con la ayuda del tercer investigador, se dirimió la controversia. Los análisis fueron presentados a cuatro participantes quienes expresaron su acuerdo con la información analizada. Con esto se dio cumplimiento a los principios de reflexibilidad, auditabilidad y credibilidad.

\section{Consideraciones éticas}

La participación fue voluntaria, se mantuvo el anonimato y la confidencialidad de los participantes mediante la asignación de un código con números y letras. La etapa de recolección de datos se inició después de que la investigación fue aprobada por el Comité de Ética de Investigación, con la firma del consentimiento informado, conforme al Reglamento de la Ley General de Salud en Materia de Investigación para la Salud de México(22). 


\section{Resultados}

El estudio se realizó con 46 informantes, el mayor porcentaje mujeres con edad promedio de 21 años (DE $\pm 2,8)$; la mayoría solteras, $26 \%$ tiene hijos; $30 \%$ trabaja además de estudiar; $91 \%$ utiliza el transporte colectivo, y sólo $3 \%$ usa otro medio para trasladarse a la institución educativa (Tabla 1).

Tabla 1. Datos sociodemográficos, según estudiantes de Licenciatura en Enfermería, México 2019

\begin{tabular}{lr}
\hline \multicolumn{1}{c}{ Variable } & N (\%) \\
\hline Edad & $21 \pm 2,8$ años \\
Mujeres & $40(87)$ \\
Hombres & $6(7)$ \\
Solteros & $38(83)$ \\
Casados & $8(17)$ \\
Con hijos & $12(26)$ \\
Trabaja y estudia & $14(30)$ \\
Total & $46(100)$ \\
\hline
\end{tabular}

Los resultados emergieron del proceso continuo en la fase de organizar, procesar y analizar el contenido latente de la abstracción e interpretación de los relatos, reflejo de la relación dialógica de su formación: la teoría (ideal) y la práctica (real). Los resultados se estructuran de acuerdo con las temáticas de los relatos: 1) El cuidado ideal, desde el mundo real; 2) La vulnerabilidad de la vida cotidiana y la formación; 3) El cuidado en un ritmo de vida distante del cuidado.

\section{El cuidado ideal, desde el mundo real}

Las narraciones dejan ver la comprensión del cuidado como una relación dialógica de su formación, para guiar su cuidado como algo existente dentro de su cotidiano, eso permite construir y transformar su ser y hacer en su día a día. Los participantes buscan descubrir su problemática social desde el mundo real, dejan lo ideal para el otro, aunque todo esto representa una línea de enseñanza que guía su cuidado, descuidan su salud humana.

«...El conocimiento ayuda y lo real ante lo ideal, pero la carga de trabajo es bastante estresante y también estar de pie muchas horas no permite cuidarte; entonces creo que la carga de trabajo desde mi rol de estudiante, no puedo comer a mi hora ni dormir las horas que debo dormir, ejercitarte, ¿a qué hora? conoces lo que implica el cuidado, pero no hay tiempo...» (E1, E37).
«...El conocer cómo cuidar la salud es teórico, pero hay sobrecarga de trabajo, eso obedece o no, según sea el caso, luego entonces, te dirige, con herramientas para cuidar, así no puedo cuidar de mí mismo...» (E2, E 45).

Es evidente que el cuidado deja de ser algo ideal para volverse real, en el momento en que los actores sociales se posesionan frente a las vivencias significativas, pero a la vez alarmantes, al dejar que su ser social viva esa carga de trabajo, más allá de sus fuerzas, a pesar de ser conscientes de no cuidarse como ser humano que vive y siente, lo descuidan desde estas epatas tempranas de la vida profesional.

\section{La vulnerabilidad de la vida cotidiana y la formación}

De acuerdo con el discurso de los estudiantes de enfermería, ubican el cuidado como una acción habitual donde se apropian del conocimiento para transformar, y construir un cuerpo de saberes de la acción de cuidado; desde su cotidiano, perciben la salud que se revierte y es más un reto transformar el ser de cuidado de enfermería como el ser que cuida; comprender el comportamiento cotidiano de este grupo de estudiantes representa un progreso que orienta la perspectiva de la construcción del significado humano, con una base esencial para promover la salud en la vida diaria.

«...Mi formación de enfermería ha modificado mi cuidado cotidiano, tengo bases científicas para mantenerme saludable; sin embargo, los horarios, las tareas no me permiten hacer ejercicio, disfrutar de una comida saludable...» (E5).

«...La formación me da conocimientos para modificar hábitos y mantener el cuidado de nosotros mismos, no los incluimos como práctica cotidiana en nuestra vida, lógicamente no podemos cuidar a otros, pero la realidad es otra, el horario, tareas, no hay tiempo para comery mucho menos para relajarte y todo eso; eso me mueve, me hace vulnerable...» (E6).

Por lo tanto, las expectativas para mejorar la noción de los procesos de cuidado en su cotidiano se modifican por las prácticas clínicas, desde el imaginario social, en el que no se reduce la complejidad de las prácticas clínicas, en sintonía con el escaso apoyo para vencer sus propias dificultades al momento de cuidar su salud. La representación del cuidado está vinculada con el tiempo para el propio cuidado, exclusivamente como normativa de las dimensiones discursivas y textuales que se 
entrecruzan en todo cuidado, de acuerdo con lo expresado por este grupo de alumnos.

\section{El cuidado en un ritmo de vida distante del cuidado}

Los relatos muestran las experiencias en el ritmo de vida de los estudiantes de enfermería sobre el cuidado; de cierta forma, relacionados con sus necesidades de cuidado, contemplan un ritmo de vida difícil de enfrentar por el mismo rol de su formación profesional; así lo muestran los siguientes planteamientos:

«...Te acostumbras a llevar un ritmo de vida, es difícil decir tal hora hago tal cosa o actividad para mi cuidado, no puedo, simplemente, no es posible, este ritmo de vida acelerado, la escuela, las tareas, las prácticas, mi pareja, no hay tiempo para el cuidado de sí mismo...»(E10).

«...Sí, de alguna manera el ritmo de vida altera mi cuidado, sabemos cómo cuidarnos y qué requiere nuestro cuerpo para cuidarnos (E34). Muchas veces tienes que ignorar el dolor, el temor, el hambre, o la comida, no es saludable, es evidente, tenemos el conocimiento, y resulta muchas veces no lo hacemos...»(E11).

Es así como el cuidado del alumno universitario aparece reiteradamente, cuando al incorporarse a la comunidad académica, hay evidencias de un proceso de cuidado gradual y paulatino. Al introducirse al mundo de las prácticas clínicas, en los alumnos se advierte su valor de formación y deseo de saber para su ser social emergente, pero urgente.

\section{Discusión}

Es evidente, la representación social de cuidado del estudiante generada a partir de una estructura de la dialógica reflexiva(23), donde discurre el cuidado desde la formación; en ese contexto se transforma la vida cotidiana y avanza a un ritmo de vida para el cuidado; aquí se adopta de algún modo el fundamento científico, emergiendo un estado de conciencia ${ }^{(2,23)}$ y de conocimiento para simbolizar sus experiencias en significados de cuidado, a fin de desarrollar un vínculo con dicha representación social de cuidado. De acuerdo con el marco conceptual utilizado para el análisis de esta investigación, Moscovici $(4,10)$ afirma que el reflejo del ritmo de vida en momentos de crisis y las condiciones cotidianas pueden ayudar en la construcción de las representaciones sociales, en este sentido para los estudiantes, la representación social no sólo determina las acciones sobre el cuidado que realizan, sino que pueden también producir nuevos comportamientos, así como diseñar y producir nuevos comportamientos en relación con el objeto de representación. Pues como evidencian los hallazgos, el cuidado en dicha etapa de estudiantes adquiere una connotación ontológica y epistémica del hacer de enfermería $(23,24)$.

En los participantes se evidenció que, cuidar de sí, sin dejar al otro, representa una responsabilidad que necesita ser pensada y comprendida, por los riesgos implícitos en el cuidado de estos futuros profesionales de la salud(17,18), conscientes cada vez más de ese compromiso social; es evidente su fragilidad al mundo en su hacer cotidiano(2,24). Otros autores reconocen que para cuidar del otro, el estudiante de enfermería necesita pensar y comprender la situación de cuidado desde su propia vivencia y lo que significa para este cuidar $(25,26)$.

Siappo et al.,(9) y Ceballos et al.,(19) afirman que la formación del estudiante implica reflexiones de cuidado por parte de la academia. En este sentido, en los ambientes de enseñanza se deben tener en cuenta las situaciones contextuales del estudiante, por ejemplo, las escasas horas de sueño, falta de cuidados preventivos a la salud, y el sedentarismo son prevalentes durante el desarrollo de la carrera; y en consecuencia justifican, desde el punto de vista de los estudiantes, la falta de tiempo para el cuidado de sí(22,27).

Al descubrir la riqueza de las realidades sociales de las prácticas de cuidado $^{(3,28)}$ aun con las cargas emocionales $(29,30)$, se dificulta la toma de decisiones conscientes de su cuidado(2,29). En esa misma línea, se representa el desafío para mantener una mentalidad dialógica del cuidado humanizado donde se dignifique el cuidado, desde los deseos de protección y despertar la conciencia de cuidado $(4,31)$.

Por otro lado, la conciencia de cuidado, como modo de preservar la propia existencia para transformar el ser y propiciar condiciones a partir del rol de estudiante(22,30), es donde divide sus tareas entre las necesidades de salud y las actividades inherentes a la formación profesional(28), a fin de hacer un juicio clínico de cuidado cotidiano. En este sentido, la representación social del cuidado, de acuerdo con los participantes, se manifiesta por la necesidad de tiempo para cuidar(1,31), esta postura enfatiza dicha 
actividad, no como una simple tarea, que no puede medirse en una investigación, cuidar es un acto instrumental expresión del ser humano como unidad social( 5,30$)$.

La construcción del cuidado resulta de la interacción intersubjetiva entre las acciones de reflexión y los actores sociales ${ }^{(4)}$ que están a cargo del cuidado de la vida (26). Los cambios en la representación social del cuidado requieren entonces una preocupación constante por la búsqueda de autonomía y de libertad, esto favorecerá en los actores sociales una mejor visión del mundo, una manera de ser y hacer(2,12); lo que finalmente reindinvicará el acto de cuidado en medio de contextos adversos que se experimentan en la actualidad(32).

Estos procesos sociales de formación, son la esencia para experimentar diversas posibilidades para aproximarse a los conflictos sin resolver, debido a la sobrecarga de trabajo, mismas que conducen a un estado de estrés ${ }^{(2,24)}$. Ante esta situación, la ideología de las representaciones sociales de los alumnos muestra la necesidad de cuidado que se entrelaza y refuerzan su formación profesional vinculada al principio de transformación social para su cuidado, pero también, confronta la confianza del cuidado al otro; a partir de esta afirmación, se espera que ellos transformen, construyan una entidad conceptual; desde el reto más inminente de representar el ser de cuidado, como el ser que cuida y se cuida(2,22).

Se puede entender entonces la representación social del cuidado, desde el discurso del rol del estudiante ${ }^{(6,29)}$ y su forma de apropiación del cuidado. Estas formas de expresión rescatan las concepciones desde la vivencia del estudiante de su práctica del cuidado; en la que se evidencian la tensión por satisfacer las necesidades sociales actuales ${ }^{(15)}$ para un cuidado comprometido y de calidad(4,30); y la necesidad de incorporar una visión emergente donde se contenga el ideal teórico y lo práctico ${ }^{(3,22)}$, con el fin de favorecer su propio cuidado ${ }^{(3,29)}$ y el cuidado del otro, desde la dimensión social, ética y espiritual(22,26).

\section{Conclusiones}

El estudio permitió dimensionar la representación social del cuidado, como un significado para la sobrevivencia ante los procesos de su formación profesional entre acciones y reflexiones como productores del ser, y hacer consigo, acción que orienta sus conductas para cuidar del otro; a partir de las bases filosóficas. Representa el sentido a una realidad coherente con su comportamiento, y que guía el cuidado para crear estereotipos hacia una apropiación individual social, resultado del conocimiento en sus contextos dinámicos $\mathrm{y}$ cambiantes para cuidar al otro.

El comportamiento cotidiano de este grupo de estudiantes representa un progreso al orientar la perspectiva del significado humano, con una base filosófica para reiterar la importancia de promover el cuidado ideal a lo real, que sirve de referencia como futuro trabajador profesional, además de proponer estructuras curriculares innovadoras, más flexibles y menos disciplinares -con el fin de ofrecer a los estudiantes tiempo libre en sus planes de estudio, y lograr una mejor gestión de la tríade vivir/estudiar/trabajar-, deben ser valoradas en forma continua y sin riesgo para la vida humana.

Las limitaciones de este estudio podrían estar condicionadas por la experiencia de los participantes y el contexto donde se desarrolló de forma preliminar, con el fin de ampliar los resultados con mayor profundidad en estudiantes mexicanos de enfermería, de otras latitudes, así como fortalecer los estudios multicéntricos o bicéntricos y delinear nuevas líneas de investigación.

Conflicto de intereses: Ninguno declarado por los autores.

\section{Referencias}

1. González Escobar DS. Significado del cuidado para estudiantes y profesores del Programa de Enfermería de la Universidad Francisco de Paula Santander. Investig Enferm. Imagen Desarr. 2014;17(1):77-95. DOI: 10.11144/Javeriana.IE17-1.scep.

2. García-Carpintero BE, Siles-González J, Martínez-Roche ME, Martínez-Miguel E, Manso-Perea C, González-Cervantes S, et al. Percepciones de los estudiantes sobre sus vivencias en las prácticas clínicas. Enfermería Universitaria. 2019;16(3):25968. DOI: 10.22201/eneo.23958421e.2019.3.712.

3. Stein-Backes DS, Stein-Backes MS, Lorenzini-Erdmann AL, Büscher A, Salazar-Maya AM. Significado da prática social do enfermeiro com e a partir do Sistema Único de Saúde brasileiro. Aquichan. 2014;14(4):560-70. DOI: 10.5294/aqui.2014.14.4.10.

4. Moscovici S. Representações sociais. Investigações em psicologia social. Rio de Janeiro: Vozes; 2010.

5. Jodelet D. La representación social: fenómenos, conceptos y teoría. En: Moscovici S. Psicología Social II. Barcelona: Editorial Paidós; 1986.

6. Mendieta-Izquierdo G, Gómez Caicedo HE, Rojas Caucali CF. Representación social de la práctica clínica de cuidado intensivo en estudiantes de terapia respiratoria. Educ Med. 
Super. 2015;29(4):777-89. Disponible en: http://scielo.sld.cu/scielo.php?script=sci_arttext\&pid=S086 4-21412015000400011\&lng=es

7. Parreira Mendes FR, Brites Zangão MO, Parreirinha Gemito ML, Cascalho Serra I da C. Social Representations of nursing students about hospital assistance and primary health care. Rev Bras Enferm. 2016;69(2):343-50. DOI: 10.1590/00347167.2016690218i.

8. Blum CA. Practicing Self-Care for Nurses: A Nursing Program Initiative. Online J Issues Nurs. 2014;19(3):3. DOI: 10.3912/OJIN.Vol19No03Man03.

9. Martínez Rodríguez L, Venceslao Pueyo M, Ramió A. La crisis de cuidados: Percepciones del cuidado en los estudiantes de enfermería. Psicoperspectivas. 2016;15(3):135-45. DOI: 10.5027/psicoperspectivas-Vol15-Issue3-fulltext-777.

10. Gorriti Siappo CL, Rodríguez Núñez Y, Evangelista Cabral I. Nursing students' experiences in selfcare during training process in a private university in Chimbote, Peru. Esc. Anna Nery. 2016;20(1):17-24. Disponible en: https://www.redalyc.org/articulo.oa?id=127744318003

11. Duarte NE, Ferreira MA, Lisboa MTL. The practical dimension in nursing care: social representations of nursing students. Esc. Anna. 2012;16(2):227-33. DOI: 10.1590/S1414-81452012000200003.

12. Muñiz-Granoble GJ, García-Martínez MA, Rodríguez-Orozco CL, Sánchez-Hernández CA, Sandrea-Toledo LB, RebolledoMalpica DM. Competencias del ser y hacer en enfermería: revisión sistemática y análisis empírico. Rev Cubana Enferm. 2019;35(2). Disponible en: http://revenfermeria.sld.cu/index.php/enf/article/view/3 339

13. Cabral Schveitzer M, Campos Pavone Zoboli EL, da Silva Vieira MM. Nursing challenges for universal health coverage: a systematic review. Rev. Latino-Am. Enfermagem. 2016;24:e2676. DOI: 10.1590/1518-8345.0933.2676.

14. Uriarte S, Ponce G, Bernal M. Vivencias cotidianas en espacios clínicos del estudiante de enfermería. Enferm. Univ. 2016;13(3):171-7. DOI: 10.1016/j.reu.2016.07.002.

15. Crafoord M-T, Mattsson J, Fagerdahl A. Operating room nurses' perceptions of the clinical learning environment: a survey study. J Contin Educ Nurs. 2018;49(9):416-23. DOI: 10.3928/00220124-20180813-07.

16. Quiroz Reyes JA, Rivas Rivero E. Representaciones sociales del rol de la enfermera chilena en salud mental y psiquiatría 1960-1975: Una mirada histórica. Cultura de los Cuidados. 2019;23(54):171-81. DOI: 10.14198/cuid.2019.54.15.

17. San Rafael GS, Siles GJ, Solano-Ruiz C. El diario del estudiante de enfermería en la práctica clínica frente a los diarios realizados en otras disciplinas: una revisión integradora. Aquichan. 2014;14(3):403-16. DOI: 10.5294/aqui.2014.14.3.10.

18. Jiménez-Ruiz I, Almansa Martínez P. La investigación cualitativa ante las Prácticas Tradicionales Perjudiciales. Index Enferm. 2017;26(1-2):5-6. Disponible en: http://scielo.isciii.es/scielo.php?script=sci_arttext\&pid=S11 32-12962017000100001

19. Ceballos Ramos LM, Rodríguez Flores LA, González Corrales SC. La metodología de la investigación cualitativa como necesidad en la carrera de licenciatura en enfermería. Rev. Mendive. 2018;16(3):470-83. Disponible en: http://scielo.sld.cu/scielo.php?script=sci_arttext\&pid=S181 5-76962018000300470

20. Arias Valencia MM, Giraldo Mora CV. El rigor científico en la investigación cualitativa. Investigación y Educación en
Enfermería. 2011;29(3):500-14. Disponible en: http://www.redalyc.org/articulo.oa?id=105222406020

21. Martínez-Salgado C. El muestreo en investigación cualitativa: principios básicos y algunas controversias. Cienc. Saúde Colectiva. 2012;17(3):613-9. DOI: 10.1590/S141381232012000300006.

22. CÁMARA DE DIPUTADOS DEL H. CONGRESO DE LA UNIÓN. Reglamento de la Ley General de Salud en Materia de Investigación para la Salud. 2014. México. Disponible en: http://www.diputados.gob.mx/LeyesBiblio/regley/Reg_LG S_MIS.pdf

23. Fuentes-Pumarola C, Ballester-Ferrando D, Gelabert-Vilella S, Bosch-Farré C, Malagón-Aguilera MC, Rascón-Hernán C, et al. Nursing student and professor perceptions and assessments of the achievement of practicum competencies: A mixed method approach. Nurse Educ Today. 2016;45:199205. DOI: 10.1016/j.nedt.2016.08.013.

24. dos Santos ÉI, Tosoli Gomes AM, Corrêa Marques S, de Souza Ramos R, Santos AC, de Oliveira FT. Estudio comparativo sobre las representaciones de la autonomía profesional elaboradas por estudiantes iniciantes y concluyentes del curso de enfermería. Rev. Latino-Am. Enfermagem. 2017;25:e2927. DOI: 10.1590/1518-8345.1919.2927.

25. Shivers E, Hasson F, Slater P. Pre-registration nursing student's quality of practice learning: Clinical learning environment inventory (actual) questionnaire. Nurse Education Today. 2017;55:58-64. DOI: 10.1016/j.nedt.2017.05.004.

26. Sebold LF, Kempfer SS, Radünz V, Prado ML, Tourinho FSV, Girondi JBR. Cuidar é... percepções de estudantes de enfermagem: Um olhar heideggeriana. Esc. Anna Nery. 2016;20(2):243-7. Disponible en: https://www.scielo.br/j/ean/a/zkTCZPqnDqh3x9zGRVdpR Yg/?lang=en\&format=pdf

27. Celma-Vicente M, López-Morales M, Cano Caballero-Galvez MD. Analysis of clinical practices in the Nursing Degree: Vision of tutors and students. Enfermería Clínica. 2019;29(5):271-9. DOI: 10.1016/j.enfcle.2018.04.006.

28. García Reyes J, Piña Osorio JM, Aguayo Rousel HB. Representaciones sociales sobre la persona con discapacidad de estudiantes de nivel superior en México. Revista Iberoamericana De Educación. 2019;80(2):129-50. DOI: 10.35362/rie8023249.

29. Silva Ferreira E, de Souza M, de Oliveira Souza NV, Assis Tavares KF, da Silva Pires A. The importance of self-care for nursing professionals. Cienc Cuid Saude. 2015;14(1):978-85. DOI: 10.4025/cienccuidsaude.v14i1.23360.

30. Jiménez Arrollo V, Rangel Flores YY. Las representaciones sociales como marco para comprender las respuestas humanas en el cuidado enfermero. Cultura de los Cuidados. 2017;21(49):147-54. DOI: 10.14198/cuid.2017.49.16.

31. Souza M, Souza N, Tavares K, Veiga Madriaga LC. Nursing residents of intensivist scenarios: the importance of selfcare. Journal of Nursing UFPE. 2017;11(4):1634-40. Disponible en: https://redib.org/Record/oai_articulo1353228-nursingresidents-intensivist-scenarios-importance-selfcare

32. Pulido-Martos M, Augusto-Landa JM, Lopez-Zafra E. Estudiantes de enfermería em prácticas clínicas: el rol de la inteligencia emocional en los estresores ocupacionales y bienestar psicológico. Index Enferm. 2016;25(3):215-9. Disponible en: https://scielo.isciii.es/scielo.php?script=sci_abstract\&pid=S 1132-12962016000200020 